\title{
INTENSIFICAÇÃO DO USO DE FERRAMENTAS COMPUTACIONAIS NOS CURSOS DE GRADUAÇÃO EM ENGENHARIA QUÍMICA - A EXPERIÊNCIA DA UFES, CAMPUS ALEGRE
}

\author{
G. T. PELISSON ${ }^{1}$, F. T. VIEIRA ${ }^{1}$
}

${ }^{1}$ LAMCES - Laboratório de Métodos Computacionais, Controle e Estimação Universidade Federal do Espírito Santo - Engenharia Química (campus Alegre) e-mail para contato: gabriela_tosip@hotmail.com

\begin{abstract}
RESUMO - Na Engenharia Química (EQ), os programas computacionais têm sido cada vez mais utilizados. Porém, muitas vezes os estudantes não têm muito interesse e/ou não são devidamente estimulados a aplicar linguagens computacionais na solução de problemas de engenharia, algo tão fundamental na atualidade. Assim, percebeu-se a necessidade da criação de um material didático para facilitar e incentivar a implementação de meios computacionais, desde o ciclo básico, em problemas vistos em sala de aula, criando uma familiaridade e continuidade do uso de softwares como o Matlab, amplamente utilizado no ensino e pesquisa no meio acadêmico, para efetuar desde contas simples até na aplicação de métodos numéricos na solução de problemas na área de $\mathrm{EQ}$.
\end{abstract}

\section{1 - INTRODUÇÃO}

O modelo tradicional de ensino adotado na educação em Engenharia está apoiado na transmissão de conhecimentos, que normalmente focaliza os aspectos conceituais das diversas teorias, sem a sua necessária contextualização. A reprodução desses conhecimentos é valorizada por meio do estímulo à memorização, pela prática repetitiva dos mecanismos e da lógica de funcionamento dos modelos conceituais e pela aplicação de técnicas e métodos como forma única de solução de problemas (Bazzo, 1998).

É de conhecimento comum entre os profissionais do ensino de engenharia que este modelo precisa evoluir, pois não cabe mais em uma sociedade onde o conhecimento, principalmente em termos de conteúdo, pode ser adquirido por diferentes meios, além daquele transmitido pelo professor (Silveira, 2005). Atualmente, o desenvolvimento das ferramentas computacionais possibilita a expansão de uma fronteira até então pouco explorada, a da incorporação da simulação numérica como método de ensino (Belhot et al., 2001).

A modelagem matemática, em conjunto a simulação de processos são poderosas ferramentas para o estudo e entendimento do comportamento de processos complexos comuns na EQ. Estas ferramentas já encontram grande aplicação no meio industrial e no campo da pesquisa, porém ainda não são muito utilizadas no meio acadêmico como um método sistematizado para o ensino. 
Nesse sentido, inicialmente realizou-se uma enquete com o corpo discente acerca das principais demandas e implicações do uso de ferramentas computacionais para o processo de ensino/aprendizagem. O desafio desse projeto é incentivar o uso e revelar a importância da aplicação de ferramentas computacionais na solução de problemas de EQ, compreender as dificuldades de implantação de ferramentas computacionais e incentivar todo o corpo docente para o uso como recurso didático nas aulas do curso de graduação em EQ da UFES, campus Alegre.

\section{2 - APOSTILA DE MATLAB}

O projeto consiste em elaborar um material didático composto de tópicos envolvendo operações básicas em matlab, a aplicação de ferramentas na solução de problemas de EQ e também exemplos de implementação de métodos númericos, com códigos comentados linha a linha. Este é o grande diferencial desse material didático: facilitar essa tarefa, que muitas vezes é a grande barreira a ser vencida pelos alunos: como passar o método do papel para a linguagem computacional.

Os principais tópicos explorados no projeto até o momento, serão apresentados a seguir.

\section{1 - Operações com Vetores e Matrizes}

Vetores Simples: Baseando-se em Costa (2003), a criação de um vetor é simples, basta abrir um colchete e introduzir os valores separados por espaço ou vírgula e depois fechar o colchete:

$$
\begin{aligned}
& >>x=\left[\begin{array}{llllll}
0 & 0.25^{*} p i & 0.5 * p i & 0.75 * p i & p i
\end{array}\right] \\
& x=\begin{array}{llllll}
0 & 0.7854 & 1.5708 & 2.3562 & 3.1416
\end{array}
\end{aligned}
$$

Construção de Vetores: Para a construção de vetores com muitos termos, existem comandos que facilitam essa escrita. São eles:

- Criar um vetor que começa em zero e vai até o valor $\pi$, aumentando $0,25^{*} \pi$ por vez:

$$
\begin{aligned}
& >>x=(0: 0.25: 1) * p i \\
& x= \\
& \begin{array}{lllll}
0 & 0.7854 & 1.5708 & 2.3562 & 3.1416
\end{array}
\end{aligned}
$$

- Criar um vetor que começa em zero e vai até o valor $\pi$ com 5 elementos com o uso da função linspace:

$$
\begin{aligned}
& \begin{array}{l}
\text { linspace(primeiro_valor, ultimo_valor, numero_de_valores). } \\
x=
\end{array} \\
& \quad \begin{array}{lllll}
0 & 0.7854 & 1.5708 & 2.3562 & 3.1416
\end{array}
\end{aligned}
$$


Construção de Matrizes: Para a construção de matrizes no MATLAB utilizam-se espaço para separar elementos da mesma linha e ponto e vírgula para separar os elementos de uma linha da outra:

$$
\begin{aligned}
& >>g=\left[\begin{array}{llllllll}
1 & 2 & 3 & 4 & 5 & 6 & 7 & 8
\end{array}\right] \\
& g= \\
& \begin{array}{llll}
1 & 2 & 3 & 4
\end{array} \\
& \begin{array}{llll}
5 & 6 & 7 & 8
\end{array}
\end{aligned}
$$

Formando, no exemplo, uma matriz 2 por 4 . Se todas as linhas inseridas não tiverem o mesmo número de colunas, o MATLAB exibirá uma mensagem de erro.

Dimensão de Vetores e Matrizes: Algumas vezes é necessário saber o tamanho de uma matriz ou vetor, para isto existe as funções size e length no MATLAB:

$$
\begin{aligned}
& \begin{array}{l}
> \\
r
\end{array}= \\
& \quad \begin{array}{l}
2 \\
c
\end{array}= \\
& \quad 4
\end{aligned}
$$

Onde, 'r' é o número de linhas e 'c' o de colunas.

length retorna o número total de elementos ao longo da maior dimensão da matriz e para vetores, o tamanho do mesmo:

$$
\begin{aligned}
& >>a=\left[\begin{array}{llllll}
1 & 3 & 7 & 2 & 9 & 4
\end{array}\right] ; \\
& >>\text { length (a) } \\
& \text { ans }= \\
& 6
\end{aligned}
$$

\section{2 - Controle de Fluxo}

Como mostrado por Hanselman e Littlefield (2005) temos:

Loops FOR: O loop for no MATLAB tem a mesma função estudada na programação, eles repetem os comandos descritos um determinado número de vezes.

Loops WHILE: Ao contrário de comando for, o comando while executa os passos infinitas vezes até a especificação inicial ser alcançada. Para vetores e matrizes, o loop só termina quando todos os elementos são verdadeiros aos comandos.

Estrutura IF-ELSE-END: Quando é necessário aplicar um comando condicional, usa-se if e else onde se a o comando for verdadeiro ao programa a estrutura if é executado, caso contrário, o else será executado. O uso do else é opcional.

Estrutura SWITCH-CASE: Quando a variável deve se adequar a apenas um de vários comandos, ou seja, quando houver uma variável condicionada em relação a um mesmo argumento, aplica-se a estrutura switch-case. 


\section{3 - Arquivos M de Funções}

Segundo Vicente (2003), é um programa elaborado como arquivo M e que calcula algo desejado pelo programador, devolvendo somente o valor da resposta. Os comandos executados por esse programa ficam ocultos. Você só visualiza o que entra e o que sai, ou seja, uma função é uma caixa preta.

\section{Como escrever uma Função:}

- Linha $1 \rightarrow$ indica que o arquivo M contém uma função e especifica seu nome (que é o nome do arquivo sem a extensão.m). Essa linha também define as variáveis de entrada e saída.

- Próximas linhas $\rightarrow$ são comentários (textos) explicando o que essa função calcula.

Exemplo, calcular o fatorial de um número usando função: matlab

function fat $=$ fatorial $(\mathrm{x}) \quad \%$ definição da função segundo a lógica exigida pelo

fat $=1 ; \%$ inicializa o valor do fatorial

for $\mathrm{n}=1: \mathrm{x} \%$ inicialização do loop

fat $=$ fat*n; $\%$ permite a multiplicação que a operação fatorial exige

end $\%$ fim do loop

$\mathrm{Na}$ workspace do matlab o procedimento para o cálculo do fatorial seria o seguinte:

$\mathrm{g}=$ input('digite um numero: '); \% informa o valor que se deseja o cálculo do fatorial

$\mathrm{f}=$ fatorial $(\mathrm{g}) ; \%$ lógica para o cálculo do fatorial de $\mathrm{g}$

disp (['Este eh o resultado: ' num2str(f)]) \% estrutura para criar um texto informando o valor do fatorial

\section{4 - Ajuste Polinomial}

Comando polyfit: Este comando acha os coeficientes do polinômio que estamos procurando. Mas, para isto devemos especificar o grau do polinômio. Possui três argumentos: primeiro as coordenadas x e y, e depois o grau do polinômio.

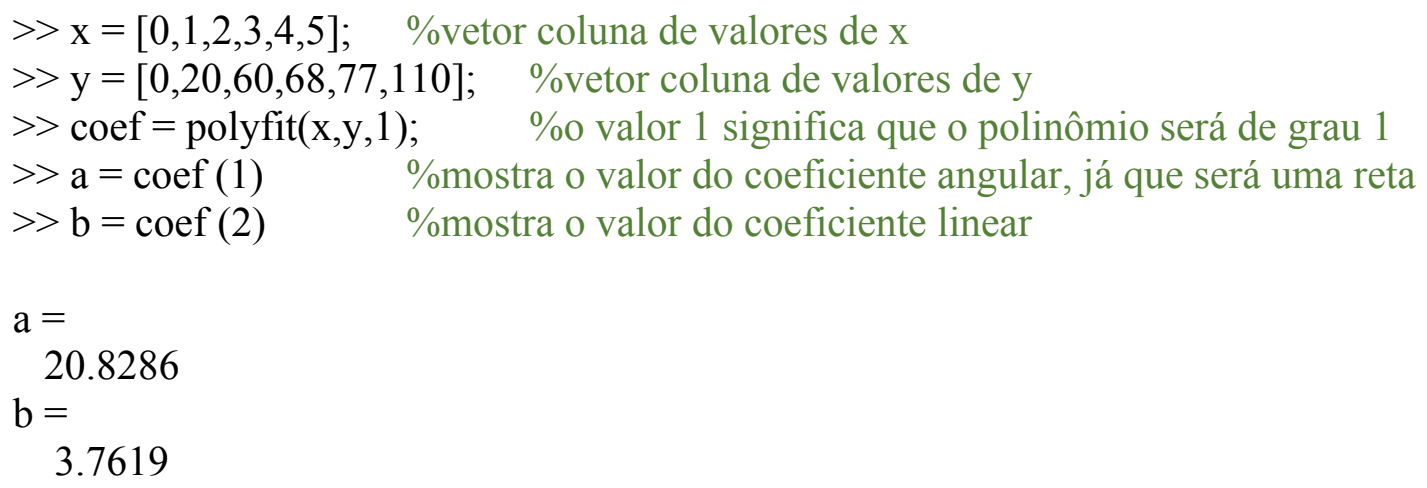


Dessa forma, teremos um polinômio da seguinte forma: $y=20,8286 . x+3,7619$.

\section{5 - Sistema Linear} $19\}$.

Considerando o sistema de equações: $\{3 \mathrm{x}+11 \mathrm{y}-2 \mathrm{z}=7 ; \mathrm{x}+\mathrm{y}-2 \mathrm{z}=4 ; \mathrm{x}-\mathrm{y}+\mathrm{z}=$

Para resolver, escreva o sistema de forma matricial $\mathrm{A} * \mathrm{x}=\mathrm{b}$, onde A é a matriz dos coeficientes, $x$ é a matriz coluna das incógnitas e b é a matriz coluna dos termos independentes:

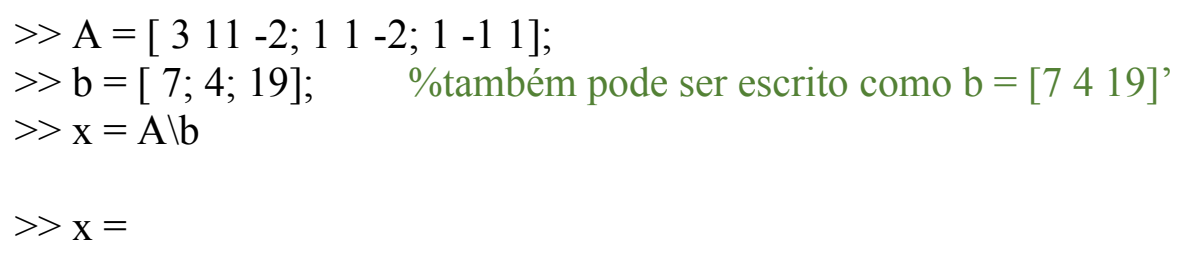

\section{6 - Um Método para Mínimos Quadrados}

É o processo que determina a função linear mais aproximada do comportamento dos pontos, calculada através do somatório dos mínimos quadrados das distâncias entre a reta e os pontos.

Um método simples que pode ser aplicado segundo Beers (2007), é a partir dos n dados das diferentes $\mathrm{p}$ incógnitas e os $\mathrm{n}$ resultados obtidos em cada 'experimento', fazer o seguinte procedimento a partir da forma genérica: $y^{k}=\beta_{0}+\beta_{1} \cdot x_{1}{ }^{k}+\beta_{2} \cdot x_{2}{ }^{k}+\ldots+\beta_{p} \cdot x_{p}{ }^{k}$.

Com $\mathrm{k}=1,2,3, \ldots, \mathrm{n}$. Dessa forma, teremos $\mathrm{n}$ equações, cada uma com os valores de um 'experimento'. Para a solução do problema, devemos descobrir os valores dos $\beta$, para chegarmos à equação linear que satisfaz os pontos dados. Teremos:

$$
Y=\left[\begin{array}{c}
y^{1} \\
y^{2} \\
\vdots \\
y^{n}
\end{array}\right] \quad X=\left[\begin{array}{ccccc}
1 & x_{1}{ }^{1} & x_{2}{ }^{1} & \ldots & x_{p}{ }^{1} \\
1 & x_{1}{ }^{2} & x_{2}{ }^{2} & \ldots & x_{p}{ }^{2} \\
1 & \vdots & \vdots & & \vdots \\
1 & x_{1}{ }^{n} & x_{2}{ }^{n} & \ldots & x_{p}{ }^{n}
\end{array}\right] \quad \theta=\left[\begin{array}{c}
\beta_{0} \\
\beta_{1} \\
\beta_{2} \\
\vdots \\
\beta_{p}
\end{array}\right]
$$

Como $\mathrm{Y}=\mathrm{X} . \theta$, para sabermos os valores de $\beta$, definida pela matriz coluna $\theta$, basta multiplicar a transposta de $\mathrm{X}$ por $\mathrm{Y}$, como no programa abaixo:

$$
\begin{aligned}
& >>X=[1-1-1 ; 1-0.699-1 ; 1-1-0.699 ; 1-0.699 \text {-0.699]; } \\
& >>\mathrm{Y}=\left[\begin{array}{l}
-4.6096 \\
-4.3157-4.2999-3.9988
\end{array}\right] \text {; } \\
& >>\text { teta }=X \backslash Y \\
& \text { teta }=
\end{aligned}
$$




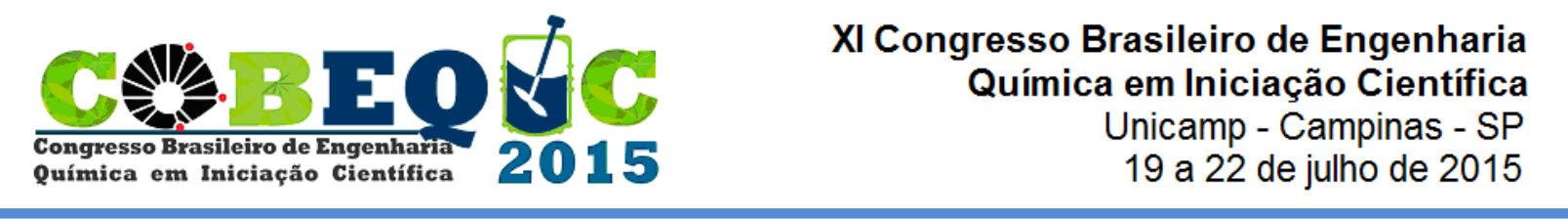

1.0409

Contudo, a equação obtida com estes resultados é $\mathrm{y}=-2,5822+0,9884 \cdot \mathrm{x}_{1}+1,0409 . \mathrm{x}_{2}$.

\section{3 - CONSIDERAÇÕES FINAIS}

Nesse trabalho é abordado apenas a parte inicial desse material. Seu desenvolvimento visa ao incentivo do uso de programas computacionais nos cursos de EQ, já que é notável a falta de traquejo da maioria dos alunos com esse tipo de ferramenta, tal essencial para o profissional na atualidade. Espera-se que, ao término da apostila, esta seja um suporte eficiente e de fácil acesso e manejo para que os alunos de EQ e outros cursos se familiarizem, desenvolvam suas habilidades e pratiquem o que foi aprendido em sala no Matlab e outros programas.

\section{4 - REFERÊNCIAS}

BAZZO, W. A. Ciência, Tecnologia e Sociedade e o Contexto da Educação Tecnológica. ed. Florianópolis: Editora da UFSC, p. 287, 1998.

BEERS, K. J. Numerical Methods for Chemical Engineering. Cambridge: Cambridge University Press, p. 373-380, 2007.

BELHOT, R. V.; FIGUEIREDO, R. S.; MALAVÉ, C. O. O Uso da Simulação no Ensino de Engenharia. Anais COBENGE 2001-CD-ROM - XXIX Congresso Brasileiro de Ensino de Engenharia. Porto Alegre: UFRGS, 2001.

COSTA, A. O. S. Matlab, Discas iniciais de utilização. Rio de Janeiro: COPPE/UFRJ, p. 6-9, 2003.

DIEGUEZ, J. P. P. Métodos e Cálculo Numérico. Rio de Janeiro: IME, p. 431, 2005.

HANSElMAN, D; LITTLEFIELD, B. Mastering Matlab 7. New Delhi: Pearson Education, p. 200-210, 2005.

SILVEIRA, M. A. A Formação do Engenheiro Inovador: Uma Visão Internacional. Rio de Janeiro: PUC-Rio, Sistema Maxwell, p. 135, 2005.

VICENTE, S. A. S. Curso introdutório de Matlab 6.5. São Paulo: Universidade Presbiteriana Mackenzie, p. 42-43, 2003. 